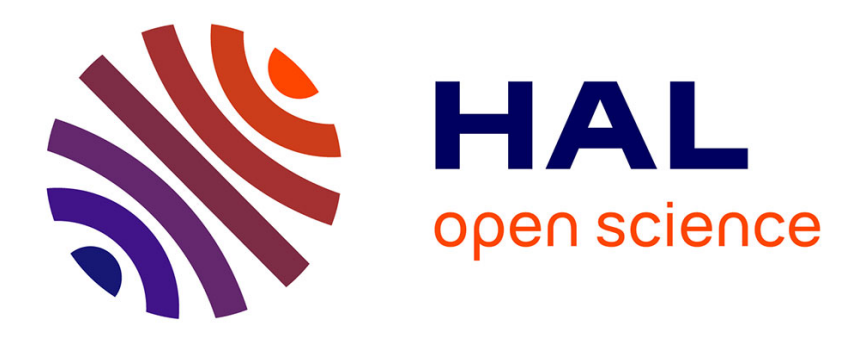

\title{
Ecrire l'histoire de la psychanalyse à l'épreuve du fragmentaire
}

Rémy Potier

\section{To cite this version:}

Rémy Potier. Ecrire l'histoire de la psychanalyse à l'épreuve du fragmentaire. Topique - Revue freudienne, 2007, Le dévoilement historique, 1 (98), pp.225-235. 10.3917/top.098.0225 . hal-01516359

\section{HAL Id: hal-01516359 \\ https://hal.science/hal-01516359}

Submitted on 14 Jul 2017

HAL is a multi-disciplinary open access archive for the deposit and dissemination of scientific research documents, whether they are published or not. The documents may come from teaching and research institutions in France or abroad, or from public or private research centers.
L'archive ouverte pluridisciplinaire HAL, est destinée au dépôt et à la diffusion de documents scientifiques de niveau recherche, publiés ou non, émanant des établissements d'enseignement et de recherche français ou étrangers, des laboratoires publics ou privés. 


\title{
Ecrire l'histoire de la psychanalyse à l'épreuve du fragmentaire
}

\author{
Rémy Potier
}

\author{
"Seule l'histoire dit à l'homme ce qu'il est », \\ Wilhelm Dilthey, Théorie des conceptions du monde. \\ Essai d'une philosophie de la philosophie \\ "Certes, nous avons besoin de l'histoire, \\ mais nous en avons besoin autrement \\ que le flâneur raffiné des jardins du savoir, \\ même si celui-ci regarde de haut nos misères \\ et nos manques prosä̈ques et sans grâce. » \\ Friedrich Nietzsche, Considérations intempestives
}

Si l'on se place sur le terrain de l'histoire des idées, la question de la scientificité de la psychanalyse fait problème. Faire problème ce n'est pas autre chose que d'inviter à la clarification, assumer les questions que pose la particularité de cette discipline, affiner un positionnement au sein d'une communauté savante, puisque de fait, la psychanalyse, au-delà de sa dimension pratique, produit un savoir qui est aujourd'hui enseigné à l'université'. Par ailleurs, la question des

1. La critique la plus célèbre qui a été faite à la psychanalyse pour remettre en cause sa prétention à la scientificité provient de Popper. La psychanalyse, contrairement aux autres sciences, produirait des hypothèses infalsifiables dans leur formulation, ce qui la cloisonnerait et ne l'inviterait pas au banquet de la marche du progrès scientifique. Cette critique est sans cesse reformulée malgré de nombreuses communications et développements sur la particularité de cette discipline qu'est la psychanalyse, sans pourtant être jamais entendu par ceux qui réitèrent cette critique, selon nous devenue aujourd'hui sans saveur. Disons que cette critique a fait son temps. Elle a été assumée et des réponses ont été apportées, si bien que pour son propre développement, la prise en compte de cette critique au sein de la communauté des analystes n'est plus de nos jours une invitation au travail. 
exigences propres à la discipline historique se pose pour l'écriture de l'histoire de la psychanalyse, car, comme l'a souligné S. de Mijolla-Mellor dans son développement, l'écriture de la psychanalyse est surtout une écriture faite de l'intérieur, le plus souvent par des psychanalystes non-historiens de formation. Il peut s'agir de prime abord d'entrer en interaction avec l'Histoire comme discipline pour dessiner les contours de l'écriture proposée par les analystes et de mettre en avant les identités et différences qui poseront les frontières dans leur justification. La question de l'écriture de l'histoire de la psychanalyse renvoie alors à une sorte d'histoire au second degré, celle de la façon d'élaborer et d'écrire son histoire, ceci a été largement montré. Aussi, ce qui se produit en la matière peut difficilement soutenir cette part d'objectivité qui fait toute la difficulté du travail de l'historien, dans la mesure où elle est bien partisane, malgré une méthode propre qui peut garantir une certaine objectivité dans sa volonté démystificatrice qui guide le travail analytique. En ce sens, il faudrait en effet attendre qu'un historien aborde la psychanalyse avec une curiosité simplement historique, en rendant certes des comptes sur sa méthode, mais surtout pour aborder son objet à travers elle, sans passion pour les figures décrites, pour les personnages évoqués, contrairement à l'écriture de l'histoire de la psychanalyse par les analystes, qui eux pourront toujours être soupçonnés d'être trop impliqués transférentiellement par rapport à leur objet. Mais ne serait-ce pas ici clore un peu vite le débat, en réservant de façon exhaustive et discutable, l'Histoire, aux seuls historiens ? La dimension historique est au cœur du travail analytique et donc de sa méthode et de sa spécificité scientifique. La question est peut-être ici davantage celle de savoir ce qui fait l'importance de l'écriture de leur histoire pour les analystes. Lacan dans la clôture du Congrès de l'EFP à Strasbourg sur inhibition et acting out précise en quelque sorte l'un de ces enjeux en relativisant ce qui doit en être l'approche :

«Bien sûr, comme tout le monde, ma réalité est faite de ceci qu'elle est ponctuelle, par rapport à ce dont on fait mémoire monumentale sous le nom d'histoire. Comme pour chacun, chacun de ceux qui sont ici et chacun de ceux qui sont aussi dehors. Il n'y a pas de ce que j'appellerai l'Histoire avec un grand $\mathrm{H}$, la grande Histoire. Il n'y a que des historioles. J'ai été pris dans une historiole qui n'est pas de moi, l'historiole freudienne, et simplement parce que j'y ai glissé. Mais il n'y a absolument rien de commun entre l'historiole freudienne et toutes celles qui l'ont précédée. Ce n'est pas parce qu'un certain nombre de gens, Herbart, Hartmann, Du Bois-Reymond, n'importe qui, ce n'est pas parce que Freud a fait ses choux gras d'un certain nombre d'épaves qui restaient des précédentes historioles que son historiole, à lui, les continue. $»^{2}$

Aussi, si la discipline qu'est l'histoire connaît ses spécificités, ses postulats

2. Lacan, Clôture du Congrès de l'EFP à Strasbourg sur inhibition et acting out, 24 mars 1976. 
et son exercice n'en restent pas moins à examiner. C'est à partir de là que l'écriture de la psychanalyse engage le positionnement de ceux qui se prêtent à l'exercice. Quelle est dès lors l'heuristique de l'histoire de la psychanalyse pour le chercheur? Comment envisager le désir d'histoire eu égard à la particularité de la psychanalyse? Quelle est la valeur des écrits historiques produits à propos de celle-ci?

Depuis ces questions, nous, apprenti-historiens, pouvons revendiquer notre pulsion de chercheur à l'endroit de l'histoire à condition de clarifier ce qui nous meut dans notre quête. Nietzsche avait bien prévenu des dangers liés à l'activité historique, en sachant en révéler corrélativement l'importance. En somme, il fait signe sur la nécessité de différencier ce qui a trait à l'instruction laborieuse, obsessionnelle et à la recherche proprement dite, stimulante et vigoureuse pour la vie. Nietzsche dégage la nécessité de l'histoire en tant qu'elle se trouve du côté de la vie. C'est à stimuler celle-ci que l'histoire peut se révéler utile, à condition que la méthodologie entreprise produise cet effet. Loin de la passion du collectionneur du savoir, la passion historique devient chez Nietzsche, généalogie. Quid alors de l'archéologue de la psyché ? La méticulosité de l'historicisme interdisant tout travail non académique ne renverrait-elle pas au problème particulier de la culture historique en faisant fi de l'apport essentiel que pourrait avoir un usage spécifique de l'histoire et de ces récits que requièrent les analystes à propos de leur discipline et de ceux qui l'ont fait vivre? Il y a bien l'idée chez Nietzsche d'un usage frauduleux de l'histoire qui en tant qu'elle contraint l'homme à s'étouffer d'un savoir pesant, sans grâce pour la faculté de l'oubli, relève d'une volonté de collectionneur plutôt que d'un désir de s'en servir pour mieux aller de l'avant. N'y a-t-il pas une leçon que la psychanalyse peut faire sienne ? Que l'on se réfère à l'attitude de Lacan opérant son retour à Freud, dévoilant par-là les conséquences d'une perte et d'un oubli sans fondement, ou au contraire, aux mimétismes que le maître à susciter sans talent et l'on aura une représentation de ce que peut-être un usage fécond et créateur des figures de l'histoire, en comparaison d'une répétition stérile et envahissante, résultant des processus identificatoires et idéalisant.

L'histoire est un récit ambigu et subversif, c'est aussi la raison pour laquelle il faut en interroger les présupposés d'une part, en en reconnaissant la nécessité d'autre part.

\section{DU DOUBLE VERSANT DE L'HISTOIRE}

Pourquoi l'écriture de l'histoire est-elle nécessaire et corrélativement pourquoi invite-t-elle à une prudence pratique liée aux exigences de toute démystification ? Les philosophes de l'histoire donnent des pistes fondamentales permettant de 
clarifier la question du positionnement à adopter dans l'exercice de cette discipline. Le problème à affronter est celui d'un idéal d'objectivité à partir des prises de consciences de la part de subjectivité dont dépend la théorie. Dilthey, par exemple, offre une réflexion qui permet d'interroger toute épistémologie et toute croyance qui ne manquent pas de porter les discours théoriques. Seulement, les analyses diltheyennes du temps historique découvrent en lui un caractère créateur : c'est un temps qui invente et innove. Seule l'histoire dit à l'homme ce qu'il est parce qu'il est un être historial et, d'autre part, c'est à travers l'interprétation de l'histoire que l'homme devient libre. Pour le philosophe, les historiens ne sont pas attentifs au fait que leurs représentations enveloppent des jugements de valeur, au lieu d'authentifier l'interprétation en y apportant un sceau d'objectivité, ils projettent inconsciemment leurs présupposés dans l'étude de l'histoire, car « même là où ils croient procéder sans présupposés, ils sont déterminés par l'horizon qui est le leur $»^{3}$

Pour illustrer concrètement ce propos et revenir au problème spécifique de l'écriture de l'histoire de la psychanalyse, je propose de partir ici de l'apport de Cornélius Castoriadis, philosophe et psychanalyste, en témoignant de l'évolution qui s'est faite jour dans sa pensée et qui peut être riche d'enseignement pour la psychanalyse au moment où elle se propose de penser sur l'écriture de son histoire. En effet, cet auteur témoigne à plusieurs titres d'un travail théorico-pratique que l'histoire met à l'épreuve en l'incitant à se clarifier. Sa pensée de l'histoire est d'ailleurs particulièrement influencée par la théorie diltheyenne. Ecrivons donc son histoire dans l'objectif avoué d'en relever quelques hypothèses. Castoriadis est né à Constantinople en 1922, il émigre en France en 1945 pour y rester jusqu'à la fin de sa vie, menant plusieurs activités, telle qu'économiste à l'OCDE, puis militant plutôt clandestin (en attente d'une naturalisation protectrice), philosophe et psychanalyste plus tardivement. Il est notable que c'est en 1968 qu'il publie pour la première fois un texte sous son nom véritable, dans la revue L'Inconscient qui deviendra Topique. C'est également le moment où il produit les concepts les plus féconds pour penser la question de l'histoire, période qui s'achemine de 1968 à 1997, année de son décès. Jacques Sédat dans son intervention comparait la position de l'analyste se penchant sur son histoire à l'embarras ressenti par le communiste à penser le communisme. Il citait à cette occasion Claude Lefort, cofondateur avec Castoriadis de la revue Socialismeoubarbarie à la fin des années 40. Or, c'est précisément pour avoir assumé cet embarras que Castoriadis me semble tout à fait intéressant pour penser la relation que les analystes entretiennent ou pourraient entretenir avec leur histoire, dans la mesure où les avancées théoriques qui résultent de cette difficile réflexion, mettent à jour une théorie de l'histoire qui rompt avec celle rependue que propose Marx, et qui pour être philosophique, n'en demeure pas moins très pertinente et stimulante pour aborder les problèmes

3. W. Dilthey, L'édification du monde de l'esprit dans les sciences humaines, 92. 
que posent l'écriture de l'histoire de la psychanalyse et son heuristique. Il faut d'emblée situer la position particulière de Castoriadis dans le champ du savoir qui peut apparaître principalement déroutante. Sa pensée se situe aux carrefours de plusieurs domaines et disciplines, comme la philosophie, la psychanalyse, la science, l'anthropologie, la sociologie, l'histoire et la politique. Or, comme point de rencontre entre plusieurs disciplines, la pensée de Castoriadis s'inscrit d'abord dans une dimension non-institutionnelle, qui trouvera tardivement sa reconnaissance de la part de l'institution en 1981 lorsqu'il est nommé à l'EHESS. Avant donc de devenir un penseur théorique institutionnellement reconnu, Castoriadis menait un militantisme important au sein de Socialisme ou barbarie. Cette période, il la consacre à l'analyse politique et économique du capitalisme ainsi qu'à l'élucidation du sens du mouvement révolutionnaire dans le monde contemporain. Ce faisant, il élabore une pensée de l'histoire conçue comme le champ du libre déploiement des forces sociales créatrices. Or, àpartirdesaréflexion sur l'histoire, pensée comme constitutive de l'action politique, Castoriadis épingle l'imagination comme un élément essentiel permettant d'appréhender la création, elle-même conçue comme surgissement du radicalement nouveau et non simple répétition du même, notamment donc à travers l'histoire. Il ne faut pas confondre la conception que Castoriadis propose de l'imaginaire avec ce que l'on connaît de Lacan sur l'imaginaire. De cela, nous allons le voir, résulte une pensée de l'histoire comme dimension originairement créatrice, ce qui s'impose néanmoins dans une rigueur aux conséquences toute méthodologiques. Ce qui est intéressant dans l'évolution de cet auteur et qui pourrait à plusieurs titres apparaître comme un exemple riche d'enseignement, c'est qu'il est d'abord résolument marxiste au moment où l'histoire contemporaine dans l'application discutable de cette doctrine incite les communistes français à s'interroger sur la pertinence de celleci. Castoriadis est concerné par les fascinations exercées par le discours marxiste, ce qui l'ouvre à la compréhension des enjeux de l'aliénation à la pensée de l'autre. Aussi dans un premier temps, son travail consiste à remettre en cause l'interprétation de Trotski, en élaborant pour autant une critique du stalinisme à partir d'un marxisme orthodoxe. Il entame progressivement un travail critique sur la pensée de Marx, pour s'en distancier définitivement dès les années 60, durant lesquelles il élabore sa pensée de l'imaginaire radical qui lui permet de concevoir l'histoire hors des cadres restrictifs du matérialisme historique, auquel, il faut le souligner, bien des historiens de métier se sont référés. Il serait d'ailleurs utile d'interroger le poids de cette conception dans bien des récits historiques, notamment à propos de la psychanalyse, en tant que celle-ci a largement fonctionné comme "opium" de l'intelligentsia. Je ne rentrerai pas dans les aspects théoriques de la doctrine de Marx que critique Castoriadis, je pointe néanmoins l'un des axes qui est la critique du matérialisme historique comme revendication d'une prétendue nécessité dans le processus historique : aucune loi dans l'histoire ne pouvait garantir qu'une révolution socialiste allait se produire et permettre 
l'avènement d'une société autogérée, d'autant moins préservée des risques de dégénérescence bureaucratique. La critique de Marx par Castoriadis propose de montrer que celui-ci n'a fait qu'extrapoler à l'ensemble de l'histoire, des schèmes de pensées propres à l'imaginaire de son époque :

«A l'interprétation vivante d'une histoire toujours créatrice du nouveau s'était substituée une prétendue théorie de l'histoire, qui en avait classé les stades passés et lui avait assigné l'étape à venir ; l'histoire comme histoire de l'homme, se produisant lui-même devenait le produit d'une évolution technique toute-puissante (...), inexplicablement progressive et miraculeusement assurant un avenir communiste pour l'humanité. $»^{4}$

Le matérialisme historique ne permettrait pas de penser l'histoire au sens fort, c'est-à-dire comme dimension des actions humaines où puissent surgir des événements nouveaux, témoignant d'une dynamique créatrice. Mais, pourrait-on m'objecter, les psychanalystes sont par nature immunisés par ces Weltanschauungen philosophiques. Il n'en reste pas moins que la croyance au progrès, dont certains récits sont porteurs, témoigne d'une conception souvent issue, même de façon latente, d'une croyance en des déterminations intrinsèques à l'histoire en marche. Cette critique pourrait tout aussi bien s'adresser à Freud, qui partageait avec l'Occident de toute son époque le postulat implicite et illusoire d'une "progressivité" immanente aux activités humaines. La première démystification majeure que propose Castoriadis, inscrit l'homme comme acteur de son histoire. Par ailleurs, rompre radicalement avec cette conception d'une histoire positive c'est également, et là l'exercice de la psychanalyse est une bonne école, se libérer de la tentation unilatérale de rationaliser le réel à outrance. Le rapport à la rationalité est bien à revisiter, elle se doit d'être à la fois fragmentaire et consciente de son aspect provisoire, il s'agit en fait d'une mise à l'épreuve à la fois fondamentale et relative. L'exercice de la psychanalyse permet de modifier son rapport à la théorie par l'écoute clinique, en sachant d'ailleurs modifier la théorie elle-même en fonction de cette même clinique. Cette dimension, l'auteur des Carrefours du labyrinthe en est conscient et il ne l'oublie pas au moment même où il philosophe. Pour lui, une théorie ne peut être achevée, au sens notamment où il est inconcevable qu'une théorie puisse atteindre le sens et la vérité de l'histoire. Il y a bien une double impossibilité d'une connaissance totale d'une institution, telle qu'en connaît la psychanalyse par exemple, et d'une anticipation rationnelle du futur de celle-ci. Mais pour Castoriadis, l'absence de savoir absolu n'est pas antinomique avec l'action consciente et délibérée, ni même d'ailleurs avec l'implication de l'inconscient dans nos vies. Il apparaît nécessaire pour le philosophe de repenser le rapport de la théorie à la pratique autrement que selon

4. Cornélius Castoriadis, La société bureaucratique, 1972, p. 46. 
le modèle traditionnel posant la primauté du penser sur le faire, différemment de Marx donc qui prisonnier de la conception hégélienne de l'histoire imposait sa vision idéaliste.

« Nous appelons praxis ce faire dans lequel l'autre ou les autres sont visés comme êtres autonomes et considérés comme l'agent essentiel du développement de leur propre autonomie." Et il ajoute, "La vraie politique, la vraie pédagogie, la vraie médecine, pour autant qu'elles ont jamais existé, appartiennent à la praxis. $»^{5}$

Je m'attarde ici à commenter cette citation pour tout enseignement pratique. Il s'agit bien de ne pas confondre la praxis avec l'articulation des moyens à une fin que l'on se serait fixée. Il s'agit plutôt de penser que l'action qui vise à la liberté des autres n'est pas étrangère à l'objet qu'elle vise, il y a bien un rapport d'intériorité entre ce qui est visé et ce par quoi il est visé :

«La praxis est, certes une activité consciente et ne peut exister que dans la lucidité ; mais elle est tout autre chose que l'application d'un savoir préalable. (...) Elle s'appuie sur un savoir, mais celui-ci est toujours fragmentaire et provisoire. Il est fragmentaire, car il ne peut pas y avoir de théorie exhaustive de l'homme et de l'histoire ; il est provisoire, car la praxis elle-même fait surgir constamment un nouveau savoir, car elle fait parler le monde dans un langage à la fois singulier et universel. $»^{6}$

La sagesse consiste donc en un bon usage du fragmentaire, considéré dans ses limites et pour les richesses qu'il contient. Ne parle-t-on d'ailleurs pas souvent trop vite de la mort de la psychanalyse comme on invoque la fin de l'histoire?

\section{LE BON USAGE DE L'HISTOIRE, ENJEU D'UNE éCRITURE}

La croyance au progrès intrinsèque à l'histoire peut être à l'origine de justification désastreuse. Aussi, l'écriture de l'histoire, fusse-t-elle celle de la psychanalyse, se doit de procéder avec la plus grande probité. D'écriture en lecteurs c'est alors l'interprétation des écrits qui tissent une représentation du passé toujours à étayer et à reprendre. Lacan met en avant cette importance de l'écrit dans l'écriture de l'histoire, ce qui fait signe vers le plaisir spécifique dont témoigne cette activité de recherche :

« Les gens écrivent au sujet de ce qui a été écrit. C'est pourquoi les documents écrits sont exigés. Vous ne pouvez faire de l'histoire

5. Cornélius Castoriadis, Institution imaginaire de la société, p. 112.

6. Ibid., p. 113. 
qu'en écrivant de seconde main sur ce qui est déjà écrit quelque part. Sans le document écrit, vous savez que vous êtes dans un rêve. Ce que l'historien exige est un texte : un texte ou un bout de papier; de toute façon, il doit y avoir quelque part, dans une archive, quelque chose qui certifie, par l'écrit, et dont le défaut rend l'histoire impossible... Ce qui ne peut être certifié par l'écrit ne peut être considéré comme de l'histoire. $\gg^{7}$

Fichte disait déjà qu'on a la philosophie de l'homme que l'on est et un soupçon significatif avait depuis été jeté sur la théorie avec Nietzsche, qui a su magistralement interroger la nécessité d'aborder l'œuvre d'un auteur en prenant en compte des éléments psycho-biographiques permettant d'éclairer d'un jour nouveau les écrits : méthode à la fois philologique et psychologique chez l'auteur de La généalogie de la morale. Cette approche spécifique du matériau historique, le « gris ${ }^{8}$, oriente le chercheur à repérer les émergences (Entstehung) pulsionnelles ; à remonter à la provenance (Herkunft) des affects, démystifiant par le même geste la prétention métaphysique à poursuivre l'origine (Ursprung) en son essence ${ }^{9}$. Ainsi, de cette pleine implication du théoricien dans sa théorie, l'exercice de la psychanalyse achève d'en convaincre. Lorsque Freud donne des repères techniques pour prévenir de l'analyse sauvage, il met bien en relief toute l'exigence critique propre à la méthodologie scientifique féconde. A ce titre, l'écriture de l'histoire de la psychanalyse ne peut que poser question, tout au moins interroge-t-elle ce qui dans l'écriture ne peut qu'être pour une part le reflet de son auteur, tout autant que celui de l'objet que celui-ci s'applique à décrire, de façon historique. Or ce qui appartient à l'auteur et ce qui relève de son objet est par nature indécidable. Il y a une sorte d'incertitude par rapport à l'histoire, une vision d'indéterminité dans laquelle on ne résout pas la question de l'histoire et dans laquelle on ne peut pas expliquer le passé et apprendre par le passé que faire. Une collectivité est capable de prendre une position au sein de laquelle le futur peut être élaboré. La vraie position humaine consiste à assumer, à accepter de prendre sur soi l'indéterminité, le risque ; connaissant qu'il n'y a ni protection ni garantie. Penser à partir de là, c'est aussi être dans la levée du refoulement et dans l'exercice de démystification des icônes et figures présentes dans l'idéal. Cette conscience a une portée méthodologique qui n'ôte rien à l'intérêt de l'histoire et de ses récits, bien au contraire. Le vœu de Castoriadis, fusse-t-il un souhait révolutionnaire, c'est que l'on passe d'une culture de la culpabilité à une culture de la responsabilité. C'est aussi que la psychanalyse est née dans ce contexte

7. J. Lacan, Yale University - Kanzer Seminar, 24 novembre 1975.

8. Nietzsche appelle le « gris » le corps livresque qu'il faut mettre à l'épreuve de la généalogie.

9. Sur les nuances des terminologies nietzschéennes pour se dégager d'une logique essentialiste et métaphysique dans son approche de l'histoire, voir Michel Foucault, « Nietzsche, la généalogie, l’histoire ». 
historique où la pensée est en quête de solution de démystification, ce que le thème de la mort de Dieu vient sonner. Prendre en compte cet événement pour penser à partir de Freud, ne consiste pas à vouloir modifier sa théorie en profondeur, ni de la refaire, mais pour notre auteur, « l'éclairer autrement à partir de ces deux thèmes qui sont restés pour elle, et non par hasard, des points aveugles : l'institution social-historique et la psyché comme imagination radicale - c'est-àdire essentiellement comme émergence de la représentation ou flux représentatifs non lié à la déterminité. ${ }^{10}$ Pour Castoriadis, la psychanalyse a encore beaucoup à faire au niveau théorique, à la fois pour explorer la psyché inconsciente et pour comprendre la relation, le pont pardessus l'abîme, qu'est la relation entre la psyché inconsciente et l'individu socialement fabriqué, ce en quoi il fait signe vers la sociologie et l'anthropologie et plus encore vers l'histoire.

Ce que nous apprend Castoriadis qui intéresse l'enjeu de l'écriture de l'histoire de la psychanalyse c'est l'importance d'une exégèse qui sans relâche articule l'individu dans le social et son histoire. Aussi, les écrits historiques ontils leur importance comme trace essentielle pour toute réflexion de fond. L'expérimentation pratique étant première sur la théorie, c'est donc aussi en acte que les textes historiques viennent modifier la psychanalyse en mouvement. L'intérêt ici de le citer en exemple, provient aussi de son expérience du travail de désidéalisation dont il témoigne dans sa vie, notamment à l'égard du marxisme. C'est alors chemin faisant que sa réflexion sur l'histoire, forte de son expérience psychanalytique, aura su épingler le risque de toute croyance exacerbée au progrès en marche. Son usage de l'histoire est plein, mais non historien dans le sens académique du terme. Il n'est pas étonnant qu'il rejoint en un sens Nietzsche lorsque ce dernier écrit que « la vie a besoin des services de l'histoire, il est aussi nécessaire de s'en convaincre que de cette autre proposition (...), à savoir que l'excès d'études historiques est nuisible aux vivants. ${ }^{11}$

Ces considérations théoriques, non dépourvues de mise en récit historique, me font rapprocher Nietzsche, Freud et Castoriadis sur un point qui est que le bon usage de l'histoire reviendrait à la méditer et à la vivre dans une activité créatrice qui sache rompre avec une illusoire continuité, mobilisant pleinement les ressorts spécifiques du présent. « Le véritable historien doit avoir la force de transformer les choses les plus notoires en choses inouïes et de proclamer les idées générales, avec tant de simplicité et de profondeur que la profondeur en fait oublier la simplicité et la simplicité la profondeur $»^{12}$. En écrivant ceci, Nietzsche met à jour une intuition dont témoigne pleinement Freud en sa découverte de l'inconscient. La leçon n'est donc autre que de savoir faire preuve d'autonomie tout en s'inscrivant dans la tradition.

10. C. Castoriadis, L'institution imaginaire de la société, p. 401.

11. Nietzsche, Considérations intempestives, II.

12. Nietzsche, Considérations intempestives, II. 
Pour finir, je condenserai le propos de Castoriadis dans la notion qu'il propose de l'autonomie, qu'il ne faut pas trop vite comprendre, mais bien méditer à partir de l'apport de la psychanalyse, car nous ne somme pas là dans les catégories métaphysiques de la tradition cartésienne.

« Je dirais qu'un individu est autonome quand il (ou elle) est vraiment en mesure de changer lucidement sa propre vie. Cela ne veut pas dire qu'il maîtrise sa vie ; nous ne maîtrisons jamais notre vie parce que nous ne pouvons pas éliminer l'inconscient, éliminer notre appartenance à la société et ainsi de suite. Mais nous pouvons changer notre relation à l'inconscient; nous pouvons créer une relation avec notre inconscient qualitativement différente de l'état où nous sommes simplement dominés par celui-ci, sans en savoir quoique ce soit. $»^{13}$

L'autonomie c'est l'autolimitation à travers la position collective de la loi. Castoriadis travaille à une solution que se passe d'un processus aliénant ou de totémisation. L'écriture de l'histoire de la psychanalyse peut jouer ce rôle si elle n'est pas apologétiste. Aussi, écrire l'histoire de la psychanalyse c'est peut-être tenter cet autonomisation de soi, en construisant son identité d'analyste en la mise à l'épreuve de ses propres icônes, qui dans l'exercice d'un idéal d'écriture peuvent enfin se dissoudre au profit du nouveau.

Rémy POTIER

42 bd de Port Royal

75005 Paris

13. C. Castoriadis, Domaines de l'homme, p. 44-45. 


\section{RéMy POTIER - ECRIRE L'HISTOIRE DE LA PSyCHANALySE À L'éPREUVE DU FRAGMENTAIRE}

\section{Rémy Potier - Ecrire l'histoire de la psychanalyse à l'épreuve du fragmentaire}

Résumé : L'enjeu de l'écriture de l'histoire de la psychanalyse s'inscrit dans la spécificité de sa scientificité. C'est peut-être pour poursuivre le travail toujours à refaire d'une démystification propre aux tendances idéalisantes que l'histoire de la psychanalyse, certes fragmentaire, peut pleinement nourrir la recherche analytique. Les particularités académiques de l'Histoire doivent également être interrogées dans leur différence, dans la mesure où l'usage que peut en faire l'analyste sert un autre idéal que celle qui meut l'historien. L'exemple de Cornélius Castoriadis peut paraître porteur pour penser l'histoire de façon concrète, loin de toute idéologie progressiste. C'est l'immanence de l'incitation à la recherche qu'en dernière analyse, l'écriture de l'histoire de la psychanalyse pourrait permettre de sans cesse relancer. La réflexion sur les conditions de possibilités d'une telle écriture ouvre dès lors à l'importance de l'investissement du fragmentaire en tant que tel. Eloge de l'inachèvement.

Mots-clés : Histoire - Histoire de la psychanalyse - Philosophie - Idéal - IdéalismeDémystification - Création - Fragmentaire.

\section{Rémy Potier - Writing the History of Psychoanalysis in the Face of Fragmentation}

Summary : The stakes at play in writing the history of psychoanalysis are part and parcel of the specificity of its scientific nature. It is doubtless with an aim to pursuing the unending task of demystifying idealising tendencies, that the history of psychoanalysis, however fragmentary it may be, manages to fuel analytical research. The academic particularities of History should also be examined in order to define their difference, as the use an analyst makes of these serves an ideal quite distinct from that which drives the historian. The example of Cornélius Castoriadis may indeed seem eloquent for us here in this consideration of history in a concrete light, devoid of all progressivist ideology. It is, after all, the very immanence of its incitement to carry out research that can be identified as the major achievement of the act of writing the history of psychoanalysis. Our consideration of the conditions that govern the possibilities of such an endeavour reveal how important our investment of these fragmentary elements as such is. The unfinished is indeed to be celebrated here.

Key-words : History - History of Psychoanalysis - Philosophy - Ideal - Idealism Demystification - Creation - Fragmentary. 